\title{
Treatment Effects of Short-Term Continuous Positive Airway Pressure on Blood Glucose Control in Type 2 Diabetic Patients with Obstructive Sleep Apnea Syndrome
}

This article was published in the following Dove Press journal:

International Journal of General Medicine

\author{
Cui Ying Wei $\mathbb{D}^{1, *}$ \\ Zhong Ming $\mathrm{He}$ (D) $^{2, *}$ \\ Han $\mathrm{Yan}^{3}$ \\ Jing $\mathrm{Li}^{3}$ \\ Pei $\mathrm{An}^{3}$ \\ Long Zhao ${ }^{3}$ \\ Li Nong $\mathrm{ji}^{4}$ \\ Zhan Cheng Gao ${ }^{3}$ \\ Xiao Song Dong ${ }^{3}$ \\ Fang $\mathrm{Han}^{3}$
}

'Department of Endocrinology, First Affiliated Hospital, Baotou Medical

College, Baotou, Inner Mongolia, People's

Republic of China; ${ }^{2}$ Department of Pulmonary Medicine, Karamay Central

Hospital, Karamay, Xinjiang, People's Republic of China; ${ }^{3}$ Department of

Respiratory and Critical Care Medicine, Peking University People's Hospital,

Beijing, People's Republic of China; ${ }^{4}$ Department of Endocrinology, Peking University People's Hospital, Beijing, People's Republic of China

*These authors contributed equally to this work

\begin{abstract}
Purpose: The study aimed at assessing glucose control measured with a continuous glucose monitoring system (CGMS) before and after short-term continuous positive airway pressure (CPAP).

Materials and Methods: Twenty-four type 2 diabetic patients (T2DM) with Obstructive sleep apnea syndrome (OSAS) (mean age $55.0 \pm 9.0$ years; BMI $29.5 \pm 5.2 \mathrm{~kg} / \mathrm{m}^{2}$ ) were admitted and kept under diet control for 2 days, then underwent 2 overnight polysomnographies: a diagnostic study and one with CPAP titration. Then they were treated by CPAP during sleep for the following three nights. Participants were divided into subgroup D (only diet control) and subgroup M (with DM medication). CGMS was utilized over the last five days. Glucose control was also assessed with plasma insulin and a clinical measure of insulin resistance (HOMA-IR) index.

Results: The mean $( \pm \mathrm{SD})$ apnea-hypopnea index (AHI) at diagnostic polysomnography was $51.2 \pm 22.4$ (range 10-88) events/h. CPAP treatment in the subjects with OSAS resulted in the index of oxygenation desaturations being reduced from $33.3 \pm 20.1$ to $1.1 \pm 1.6$ (P $=0.00$ ). CGMS showed mean 24-hours glucose values significantly lower after CPAP treatment than at baseline in both subgroups $(7.97 \pm 1.31$ vs $7.52 \pm 0.94, \mathrm{P}=0.033$ in subgroup $\mathrm{D}$; and $7.72 \pm 1.51$ vs $7.17 \pm 1.21, \mathrm{P}=0.05$ in subgroup $\mathrm{M}$ ), as the fasting plasma insulin levels and HOMA-IR were also decreased significantly after CPAP treatment $(13.0 \pm 7.5 \mu \mathrm{U} / \mathrm{mL}$ vs $10.8 \pm 5.4 \mu \mathrm{U} / \mathrm{mL}, \mathrm{P}=0.044 ;$ and $4.2 \pm 2.2$ vs $3.1 \pm 1.7, \mathrm{P}=0.003$, respectively). Standard deviation (SD) and mean amplitude of glucose excursions (MAGE) were also decreased in the subgroup $\mathrm{D}(1.91 \pm 1.10$ vs $1.61 \pm 1.20, \mathrm{P}=0.014 ; 1.26 \pm 1.13$ vs $1.01 \pm 0.98, \mathrm{P}=0.008$, respectively) only.
\end{abstract}

Conclusion: Short-term CPAP treatment in OSAS with type 2 diabetic patients is accompanied by a decrease in blood glucose level and improved insulin sensitivity. Glucose variability was reduced but only in the patients with diet control.

Keywords: type 2 diabetes mellitus, obstructive sleep apnea syndrome, continuous positive airway pressure, continuous glucose monitoring system, glucose variability, HOMA-IR

\section{Introduction}

Obstructive sleep apnea syndrome (OSAS) is associated with impaired glucose metabolism, resulting mainly from insulin resistance, with developing type 2 diabetes mellitus (T2DM), ${ }^{1-3}$ and, more recently, with the metabolic syndrome
Department of Respiratory and Critical

Care Medicine, Peking University People's

Hospital, Beijing, People's Republic of

China

Tel/Fax +86-10-88324207

Email dongxiaosong@pkuph.edu.cn
International Journal of General Medicine 2020:13 1567-1573

1567

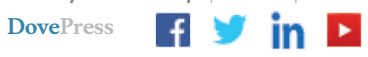

(c) (1) (2) $\odot 2020$ Wei et al. This work is published and licensed by Dove Medical Press Limited. The full terms of this license are available at https://www.dovepress.com/terms.php

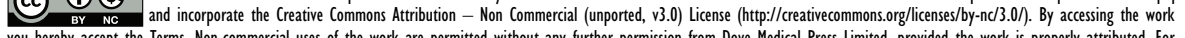
you hereby accept the Terms. Non-commercial uses of the work are permitted without any further permission from Dove Medical Press Limited, provided the work is properly attributed. For permission for commercial use of this work, please see paragraphs 4.2 and 5 of our Terms (https://www.dovepress.com/terms.php). 
in the absence of meeting T2DM criteria. ${ }^{4}$ However, data on early or late treatment effects with continuous positive airway pressure (CPAP) on blood glucose and/or insulin levels are conflicting with some reports showing no effect or worsening of values. ${ }^{5-10}$ Differences among studies are attributed to patient heterogeneity and disease treatment and/or duration, as well as to behavioral factors, sleep, and circadian rhythm. Considering both OSAS and the pathophysiology of T2DM, single point-in-time values of glucose before and after treatment might result in incomplete data and interpretations. Moreover, the variability in glucose over time might be more important since both upward and downward glucose fluctuations are potent activators of oxidative stress. Even though many methods have been proposed for assessing glucose fluctuations, the mean amplitude glucose excursions (MAGE) index is considered the "gold standard". MAGE estimation requires the use of continuous glucose sensors. We have therefore conducted a study aiming to assess the treatment effect of short-term CPAP on glucose control measured with a continuous glucose monitoring system (CGMS). ${ }^{11}$

\section{Materials and Methods}

\section{Patients}

Twenty-four patients with T2DM were originally referred by the Diabetes Unit for confirmed diagnosis of OSAHS. They agreed to participate in the study and were admitted into the ward of the sleep center. Fourteen newly diagnosed T2DM patients without anti-diabetic medication (diet only, named subgroup D) were instructed to keep a strictly-controlled diabetic diet and dietary record during the study, and the other ten patients kept their diabetic treatments without instructions to follow a different or strict diet (with medication, named subgroup M). All the patients had kept diet control as before. Exercise was not controlled, however, both subgroups remained in the ward for the duration of the study. No patient had acute cardiopulmonary illness (recent MI, stroke, etc.) or was hospitalized in the past 6 months. Patients were excluded if they were unable or unwilling to provide consent or to receive CPAP therapy. The study was approved by the ethics committee of Peking University, People's Hospital. The study was conducted in accordance with the Declaration of Helsinki and all patients gave their written informed consent to participate in the study.

\section{OSAHS Treatment Protocol}

After two days of diet control, all the patients underwent a diagnostic PSG in the third night, then on the fourth night started CPAP titration and pulse oximetry monitoring. During the following 3 nights, patients were treated with CPAP while sleeping. Treatment pressures were titrated in all patients with auto-CPAP (AutoSet Spirit, ResMed, Australia) with standard settling of 30 minutes and basal positive airway pressure of 4 or $5 \mathrm{cmH}_{2} \mathrm{O}$. Those periods with low constant pressure ("settling") were to enable a patient to fall asleep and were not included in the calculation of the apnea-hypopnea index (AHI). All CPAP pressures were obtained from the mask gauge connected to the pressure transducer in the yoke box, which was calibrated with the external CPAP device from 0 to 20 mbar. For all the patients in the study, the standard nasal CPAP was applied. After CPAP titration, the patients were treated with CPAP for the following three nights and underwent a continuous pulse oximetry in the last night.

\section{CGMS Protocol}

All the patients began continuous glucose monitoring (CGMS, MiniMed, Northridge, CA, USA) applied on the third morning (before nocturnal PSG) for overnight during the last five days of the study. As shown in Figure 1, in the third morning participants started with CGMS, and underwent PSG, titration and 3 days of CPAP treatment for a total of 5 days. In each patient, the CGMS monitor was calibrated 4 times during each night: 1 minute before and 4 hours and 3 hours after the monitor was turned on and 1 minute before it was turned off. ${ }^{11}$ Fasting plasma glucose and insulin were assessed at baseline and after the last CPAP night (ELISA, reagents from DAKO, Ely, UK; reference range 10.8-85.8 $\mathrm{pM}$ ). Flow chart shown in Figure 1.

\section{Data Analysis}

Values were grouped as the mean interstitial glucose from lights out to lights on (nocturnal), from lights on to light out (diurnal), and for the 24-hours (from 8am to 8am). The primary endpoint was the mean interstitial glucose for the mean glucose level of 24-hours. The standard deviation (SD) of the glucose level was extracted to describe glucose fluctuations. As an additional assessment of glucose variability, we calculated the mean amplitude of glucose excursions (MAGE). The homeostatic model assessment of insulin resistance (HOMA-IR) index was used to assess 
Two days of diet control

The third morning: Fasting glucose and insulin.

The third day: CGMS monitoring began (values of this day were extracted as the results of pre-treatment)

The third night: PSG study

The fourth night: CPAP titration and pulse oxymetry monitoring

The following three night: CPAP titration and treatment (CGMS values of the last day were extracted as the results of post treatment).

The last morning: fasting glucose and insulin

Figure I The flow chart of the study protocol.

each patient's insulin resistance. The HOMA-IR was calculated from fasting plasma glucose and insulin values measured in a single sample according to the formula insulin (expressed in $\mathrm{mU} / \mathrm{L}$ ) $\times$ glucose $(\mathrm{mmol} / \mathrm{L}) / 22.5$. The HOMA-IR has been repeatedly shown to be a reliable indicator of human insulin resistance (the greater the index value, the greater the insulin resistance). ${ }^{12}$

\section{Statistical Analysis}

The normal distribution test was conducted in the variables and all the index were normally distributed. The normal distribution parameters are represented as mean \pm standard deviation (SD). All statistics were analyzed with SPSS 11.5 (SPSS Inc., Chicago, IL, USA). Comparisons of pre- and post-treatment values were performed by paired $t$-tests, while comparisons of values of the two subgroups were performed by independent sample $t$-test, when a $P<0.05$ was considered significant.

\section{Results}

\section{Clinical Findings and Oxygen Saturation Data of the Patients with CPAP}

Twenty-four patients diagnosed with T2DM and OSAS were recruited into and completed the study. Their characteristics are described in Table 1. 16 of the 24 participants had a body mass index greater than $28 \mathrm{~kg} / \mathrm{m}^{2}$, and 10 had a body mass index greater than 30 . Twenty-one of the 24 had severe sleep apnea (AHI > 30).

Table I Characteristics of the 20 Men and 4 Women Who Took Part in the Study

\begin{tabular}{|c|c|c|c|c|}
\hline & All (mean $\pm S D)$ & Subgroup D (Mean $\pm S D)$ & Subgroup M (Mean \pm SD) & $\mathbf{P}$ \\
\hline Age $(y)$ & $55.0 \pm 9.0$ & $56.21 \pm 9.40$ & $54.90 \pm 9.37$ & 0.74 \\
\hline $\operatorname{Sex}(M / F)$ & $18 / 6$ & $11 / 3$ & $7 / 3$ & 0.47 \\
\hline BMI $\left(\mathrm{kg} / \mathrm{m}^{2}\right)$ & $29.5 \pm 5.5$ & $28.98 \pm 4.08$ & $29.27 \pm 5.15$ & 0.88 \\
\hline SIT90 (\%) & $12.3 \pm 13.4$ & $26.04 \pm 26.4 I$ & $14.97 \pm 15.70$ & 0.25 \\
\hline $\mathrm{AHI}(/ \mathrm{hr})$ & $51.2 \pm 22.4$ & $53.07 \pm 20.19$ & $46.89 \pm 23.30$ & 0.55 \\
\hline ODI (/hr) & $33.3 \pm 20.1$ & $33.13 \pm 16.81$ & $32.4 \pm 21.22$ & 0.93 \\
\hline HbAlc (\%) & $7.1 \pm 0.9$ & $7.00 \pm 0.69$ & $7.23 \pm 0.98$ & 0.51 \\
\hline FPG $(\mathrm{mmol} / \mathrm{L})$ & $7.97 \pm 3.05$ & $7.69 \pm 3.27$ & $8.37 \pm 2.85$ & 0.59 \\
\hline HOMA & $3.84 \pm 2.17$ & $3.89 \pm 2.51$ & $3.77 \pm 1.70$ & 0.90 \\
\hline
\end{tabular}

Note: Differences between two subgroups for each factor were evaluated and the significance levels were listed above. 
All of the major oxygen saturation parameters improved significantly on CPAP titration. The index of oxygenation desaturations (number of desaturations $\geq 4 \%$ per hour of recording time) was reduced from $33.3 \pm 20.1$ to $1.1 \pm 1.6$ $(\mathrm{P}=0.000)$. The lowest saturation value rose from $73.5 \% \pm$ $16.0 \%$ to $88.9 \% \pm 6.3 \%,(\mathrm{P}=0.000)$. The SIT $90 \%$ (the percentage of total sleep time spent below a saturation of $90 \%$ ) was reduced from $12.3 \% \pm 13.4 \%$ to $0.08 \% \pm 0.21 \%$ $(\mathrm{P}=0.002)$.

\section{Plasma Glucose, Insulin and HOMA-IR Index}

Fasting plasma insulin (FPI) levels in patients with OSAS was $13.0 \pm 7.53 \mu \mathrm{U} / \mathrm{mL}$ at baseline, and the HOMA-IR was $4.2 \pm 2.2$. Fasting plasma glucose tended to be lower after CPAP treatment than at baseline $(6.44 \pm 1.67 \mathrm{mmol} / \mathrm{L}$ vs 7.59 $\pm 2.95, \mathrm{P}=0.068$ ). Both FPI and HOMA-IR index were decreased significantly (fasting insulin was $10.8 \pm 5.4 \mu \mathrm{U} / \mathrm{mL}$, and HOMA-IR index $3.1 \pm 1.7, \mathrm{P}<0.05$ ). The changes of glucose level monitored by CGMS after CPAP treatment in a participant recruited to subgroup D are shown in Figure 2.

\section{Interstitial Glucose in CGMS}

The CGMS showed mean glucose values significantly lower with CPAP treatment than those at baseline not only during sleep but also during the whole day, while they tended to be lower during teh daytime but not significantly. However, the variability of the 24-hour glucose levels when assessed by either the SD or MAGE was decreased only during sleep (see Table 2).

The changes after CPAP treatment in two subgroups are shown in Table 3. CGMS showed mean 24-hour glucose values significantly lower after CPAP treatment than at baseline in both subgroups, the SD, MAGE and HOMA-IR were decreased significantly after CPAP treatment in subgroup D. The changes of MAGE between subgroup $D$ and subgroup $M$ is significant $(-0.27 \pm 0.68$ vs $-0.06 \pm 0.04, \mathrm{P}=0.036$ ), indicating glucose variability were reduced more in the participants with diet only.

When we compared the above changes between male and female patients, the difference is not significant. We also did not find a significant difference in the changes between patients with severe OSA (AHI $>30$ events/h) or not. The changes were significantly different between obese patients and non-obese patients in MD and HOMA. The results are shown in Table 4.

\section{Discussion}

This study showed that in patients with T2DM and OSAS, treated by CPAP for three nights: 1) in the patients with diet only or with medication treatment, the mean nocturnal glucose levels and 24-hour values were decreased $(\mathrm{P}=0.00) ; 2)$ glucose levels were less fluctuated during sleep after CPAP, but not during daytime; 3) variability

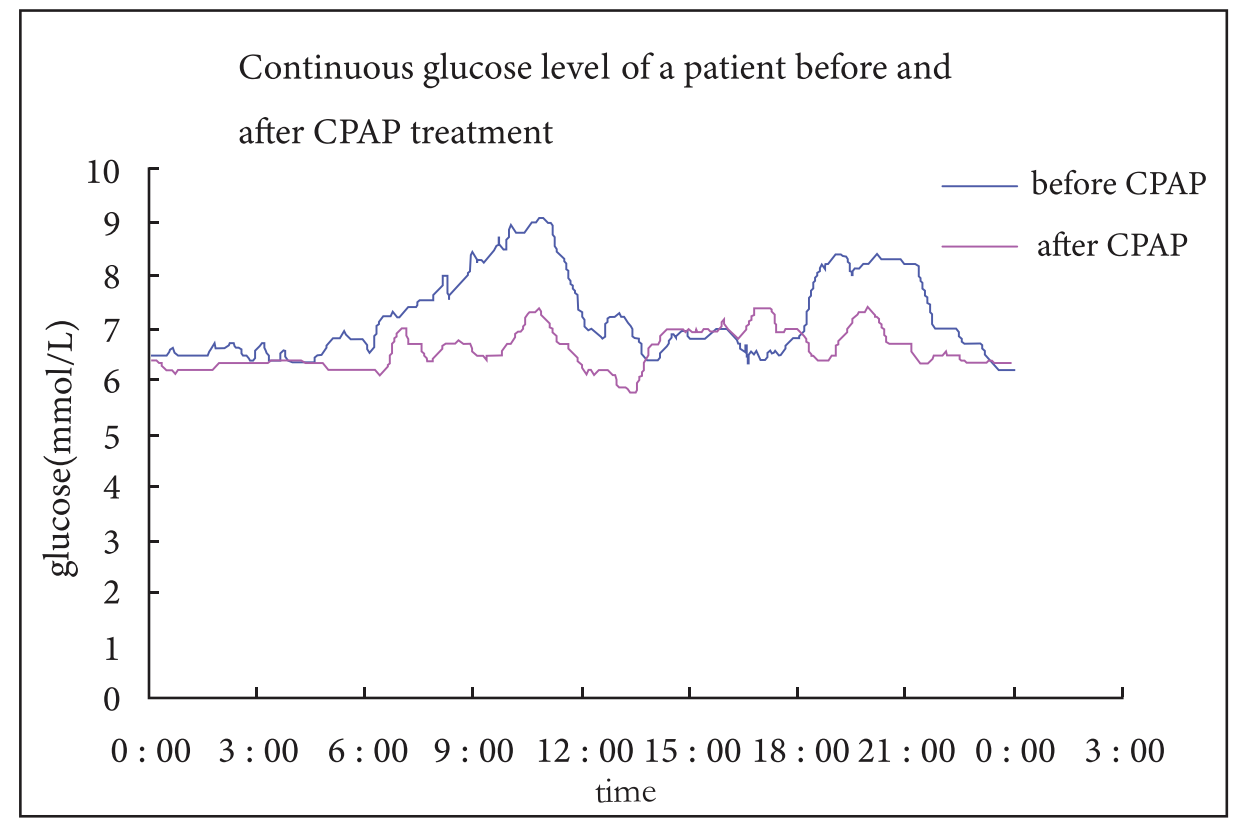

Figure 2 The changes of glucose level monitored by CGMS after CPAP treatment in subgroup D. 
Table 2 Glucose Values at Baseline and with CPAP Treatment $(n=24)$

\begin{tabular}{|c|l|l|l|}
\hline & $\begin{array}{l}\text { Baseline } \\
\text { (Mean } \pm \text { SD) }\end{array}$ & $\begin{array}{l}\text { CPAP Treatment } \\
\text { (Mean } \pm \text { SD) }\end{array}$ & P value \\
\hline $\begin{array}{l}\text { Mean Glucose } \\
\text { level (mmol/L) } \\
\text { Nocturnal } \\
\text { Diurnal } \\
\text { 24-hours }\end{array}$ & $7.7 \pm 2.6$ & $7.0 \pm 1.9$ & \\
\hline SD (mmol/L) & $8.6 \pm 2.8$ & $7.9 \pm 1.8$ & 0.01 \\
Nocturnal & $0.7 \pm 0.6$ & $0.5 \pm 0.5$ & 0.22 \\
Diurnal & $1.8 \pm 1.1$ & $1.6 \pm 0.9$ & 0.03 \\
\hline 24-hours & $1.7 \pm 1.0$ & $1.6 \pm 0.9$ & 0.05 \\
\hline MAGE (mmol/L) & & & 0.32 \\
Nocturnal & $0.4 \pm 0.4$ & $0.3 \pm 0.2$ & 0.41 \\
\hline $\begin{array}{l}\text { Diurnal } \\
\text { 24-hours }\end{array}$ & $1.4 \pm 1.3$ & $1.2 \pm 0.9$ & 0.03 \\
\hline AUC 7.8 & $1.3 \pm 1.1$ & $1.1 \pm 0.9$ & 0.21 \\
Nocturnal & $1.2 \pm 2.0$ & $0.5 \pm 1.1$ & 0.25 \\
Diurnal & $1.7 \pm 2.2$ & $1.0 \pm 1.3$ & 0.03 \\
24-hours & $1.6 \pm 2.2$ & $1.0 \pm 1.3$ & 0.10 \\
\hline Fasting insulin & $13.0 \pm 7.5$ & $10.8 \pm 5.4$ & 0.10 \\
( $\mu$ U/mL) & & $3.1 \pm 1.7$ & 0.04 \\
HOMA-IR & $4.2 \pm 2.2$ & & 0.00 \\
\hline
\end{tabular}

Note: Values are mean \pm standard deviation (SD).

Abbreviations: MAGE, mean amplitude glucose excursions; AUC7.8, area under curve glucose level > 7.8mmol/L; HOMA-IR, homeostatic model assessment of insulin resistance.

Table 3 Changes of Glucose Values of the Two Subgroups After CPAP Treatment

\begin{tabular}{|l|l|l|l|}
\hline & $\begin{array}{l}\text { Subgroup } \\
\text { D (Mean } \pm \text { SD) }\end{array}$ & $\begin{array}{l}\text { Subgroup } \\
\text { M (Mean } \pm \text { SD) }\end{array}$ & $\mathbf{P}$ \\
\hline Mean Glucose & $-1.21 \pm 2.27^{*}$ & $-0.56 \pm 0.78^{*}$ & 0.39 \\
level (mmol/L) & & & \\
SD (mmol/L) & $-0.29 \pm 1.01^{*}$ & $-0.08 \pm 0.60$ & 0.056 \\
MAGE (mmol/L) & $-0.27 \pm 0.68^{*}$ & $-0.06 \pm 0.44$ & $0.036^{\#}$ \\
HOMA-IR & $1.5 \pm 1.4^{*}$ & $0.7 \pm 1.1$ & 0.187 \\
\hline
\end{tabular}

Notes: *means the changes after CPAP were significant compared with the baseline within the same subgroup, but not between the two subgroups. \# means the difference of the MAGE change compared between two subgroups was significant.

of the 24-hour glucose levels when assessed by either the $\mathrm{SD}$ or MAGE, was decreased in the diet only subgroup, but not the medication-treated T2DM patients with OSA, and 4) the HOMA-IR index was also decreased, indicating improvement in glucose tolerance.

The significant improvement in glucose level during sleep achieved with CPAP happened over 3 days in the present study independent of the treatment with diet or medication. An immediate effect was observed by Pallayova et al, ${ }^{13}$ who reported that the mean and SD of the overnight interstitial glucose decreased rapidly, with significant improvement during a CPAP titration night. Dawson $\mathrm{A}$. et $\mathrm{al}^{14}$ found that at three months of CPAP treatment nocturnal hyperglycemia and the interstitial glucose level during sleep was less variable in a group of mostly obese T2DM with mostly severe OSA. Nearly the same, in our study, after even short-term CPAP treatment, we also found that the glucose values and variability during sleep were reduced in our patients with T2DM and OSA. Some previous research found controversial effects on glucose control in the patients with T2DM and OSA. Shaw JE et $\mathrm{al}^{15}$ found no effect of positive airway pressure therapy on glucose control (evaluated by HbAlc) in patients with relatively well-controlled type 2 diabetes and obstructive sleep apnea after 6-months of PAP treatment. Nakata and his colleagues found that severity of SDB was associated with higher GV, but DM as well as $\mathrm{HF}$ diminished the contribution of SDB to glucose variability, however treatment with CPAP was effective for reduction of glucose variability only in patients without DM but not with DM. There are several possible explanations for these findings. First, patients' life style in the short-term study would not influence the glucose control. As in our study, the in-patient setting could keep the patients strictly controlling their diet as instructed. To avoid the effect of diet control, we used the CGMS values after two days of admission. However, long-term effects of PAP therapy must be influenced by the diet control, exercise and sleep hygiene, etc. Second, long-term adherence to PAP therapy may have been insufficient to produce benefits. Indeed, the patients in our study keep on CPAP for the whole night of sleep (at least 7-8 hours per night). Nearly teh same results were found in the study of Pamidi $\mathrm{S}$ and his colleagues, which showed benefits of PAP therapy in people with pre-diabetes who had in-laboratory supervision ensured adherence of 8 hours per night. ${ }^{16}$ Third, the weight gain may obscure the effects of CPAP therapy in the long-term studies. As reported in a recent study, PAP therapy may promote weight gain, ${ }^{17}$ though the mechanism of this phenomenon is unclear.

We also found that the short-term CPAP is more effective for the treatment-naïve DM patients than for the treated DM patients, not only in regards to glucose level but also in regards to glucose variability. These different effects between different DM subgroups may be caused by the process of DM history and the level of glucose control. 
Table 4 Changes of Glucose Values of Adjusting for Gender, BMI and OSA Degree

\begin{tabular}{|c|c|c|c|c|c|c|c|c|}
\hline & $\begin{array}{l}\text { Mean Glucose Level } \\
(\mathrm{mmol} / \mathrm{L})\end{array}$ & $P$ value & SD (mmol/L) & $P$ value & MAGE (mmol/L) & P value & HOMA-IR & $P$ value \\
\hline \multicolumn{9}{|l|}{ Gender } \\
\hline Male & $-1.00 \pm 2.12$ & 0.608 & $-1.1 \pm 0.97$ & 0.594 & $-1.20 \pm 0.68$ & 0.597 & $1.07 \pm 1.33$ & 0.755 \\
\hline Female & $-0.54 \pm 0.50$ & & $-0.34 \pm 0.68$ & & $-0.28 \pm 0.36$ & & $0.86 \pm 1.08$ & \\
\hline \multicolumn{9}{|l|}{ BMI $\left(\mathrm{kg} / \mathbf{m}^{2}\right)$} \\
\hline $\mathrm{BMI}<28$ & $0.03 \pm 0.77$ & 0.086 & $0.35 \pm 0.47$ & $0.042 *$ & $0.10 \pm 0.35$ & 0.151 & $0.08 \pm 0.72$ & $0.006 *$ \\
\hline $\mathrm{BMI} \geq 28$ & $-1.34 \pm 2.07$ & & $-4.3 \pm 0.95$ & & $-0.29 \pm 0.68$ & & $1.61 \pm 1.14$ & \\
\hline \multicolumn{9}{|l|}{ AHI (/h) } \\
\hline $\mathrm{AHI}<30$ & $-2.02 \pm 3.15$ & 0.125 & $-0.7 I \pm I .74$ & 0.427 & $-0.41 \pm 1.14$ & 0.573 & $0.02 \pm 0.89$ & 0.066 \\
\hline $\mathrm{AHI} \geq 30$ & $-0.59 \pm|.3|$ & & $-0.22 \pm 0.50$ & & $-0.09 \pm 0.41$ & & $1.30 \pm 1.20$ & \\
\hline
\end{tabular}

Note: *means the differences of the changes after CPAP were significant compared between the two groups.

Harsch et $\mathrm{al}^{18}$ found modestly increased insulin sensitivity after 2 days of CPAP treatment; however, this effect was not observed at all in subjects with a body mass index $>30 \mathrm{~kg} / \mathrm{m}^{2}$. Increased insulin sensitivity was also found by Brooks et al in patients with T2DM after 4 months of CPAP treatment. ${ }^{6}$ Others have failed to note any effect of CPAP on glucose control. ${ }^{5,7,8}$ As noted by reviews on the subject, some of these studies were methodologically flawed. ${ }^{8}$ Weinstock et al $^{19}$ found that impaired glucose tolerance (IGT) did not normalize after CPAP in subjects with moderate sleep apnea and obesity. However, insulin sensitivity improved in those with AHI $\geq 30$, suggesting beneficial metabolic effects of CPAP. Although mean BMI was lower and the ethnicity different, in our present study, the HOMA-IR index was commonly decreased in T2DM patients with OSA after CPAP treatment, which suggested that even short-term CPAP treatment could improve insulin sensitivity in diabetic patients.

Even if our data suggest that daytime glucose levels did not decrease with CPAP therapy, it could have a beneficial effect on the course of T2DM by reducing the nocturnal fluctuations in the glucose level. Furthermore, in the subgroup of the newly-diagnosed T2DM patients with OSA, the glucose level and its variability during 24 hours was decreased significantly. It has been shown that variability of the blood glucose level is an independent risk factor for mortality and for retinopathy in type 2 diabetics. ${ }^{20,21}$ However, Babu et al found that, in the patients treated with CPAP, postprandial glucose levels were lower after all 3 meals, ${ }^{22}$ the differences to prior work may also be partly explained by the rather short treatment duration. We also could find the same effects of CPAP on the newly-diagnosed T2DM patients with
OSA without anti-diabetic medications, who might have shorter history of hyperglycemia.

A major limitation was the lack of a control arm, either with a parallel or crossover design. We point out that patients were on CPAP for only three nights and appeared to not change their activity behavior. However, based on this data future studies can power appropriate design and select patients better for this approach. A potential limitation is that most of our patients had severe OSA (21/24 had an AHI $>30$ events/h). CPAP may have a different effect on the glucose control in less severe patients, or alternatively more severe OSA might chronically influence glucose metabolism through sympathetic or intermittent hypoxia.

\section{Conclusion}

Our study found that in T2DM patients with OSAS, CPAP exhibits a decreasing effect on mean blood glucose and glucose variability during sleep, and an effect on waketime values is not obvious. CPAP may also improve, and rapidly so, insulin sensitivity in these patients with OSAS. Our findings provide the rationale, selection criteria, design, and power calculations for further studies including random assignment of subjects to sham-CPAP and effective-CPAP arms using this technology of continuous glucose monitoring.

\section{Acknowledgments}

This study was supported by research grants from the National Science Foundation of China (81570083) (XS. Dong) (81660020) (CY. Wei) and (81360016) (ZM. He). The abstract of this paper was presented at the 6th World Congress on Sleep Medicine, 21st to 25th March 2015, Seoul, South Korea, as a poster presentation with interim 
findings. The poster's abstract was published in "Poster Abstracts" in Sleep Medicine: Hyperlink with DOI (10.1016/j.sleep.2015.02.1446).

\section{Disclosure}

The authors report no conflicts of interest for this work.

\section{References}

1. Tiihonen M, Partinen M, Narvanen S. The severity of obstructive sleep apnoea is associated with insulin resistance. J Sleep Res. 1993;2 (1):56-61. doi:10.1111/j.1365-2869.1993.tb00062.x

2. Vgontzas AN, Papanicolaou A, Bixler EO, et al. Sleep apnea and daytime sleepiness and fatigue: relation to visceral obesity, insulin resistance, and hypercytokinemia. J Clin Endocrinol Metab. 2000;85 (3):1151-1158. doi:10.1210/jcem.85.3.6484

3. Punjabi MN, Sorkin JD, Katzel LI, et al. Sleep-disordered breathing and insulin resistance in middle-aged and overweight men. $\mathrm{Am}$ $J$ Respir Crit Care Med. 2002;165(5):677-682. doi:10.1164/ ajrccm.165.5.2104087

4. Coughlin SR, et al. Obstructive sleep apnoea is independently associated with an increased prevalence of metabolic syndrome. Eur Heart J. 2004;25(9):735-741. doi:10.1016/j.ehj.2004.02.021

5. Saini J, Krieger J, Brandenberger G, et al. Continuous positive airway pressure treatment. Effects on growth hormone, insulin and glucose profiles in obstructive sleep apnea patients. Horm Metab Res. 1993;25(07):375-381. doi:10.1055/s-2007-1002123

6. Brooks B, Cistulli PA, Borkman M, et al. Obstructive sleep apnea in obese noninsulin-dependent diabetic patients: effect of continuous positive airway pressure treatment on insulin responsiveness. J Clin Endocrinol Metab. 1994;79:1681-1685.

7. Cooper BG, White JE, Ashworth LA, et al. Hormonal and metabolic profiles in subjects with obstructive sleep apnea syndrome and the acute effects of nasal continuous positive airway pressure (CPAP) treatment. Sleep. 1995;18:172-179.

8. Smurra M, Philip P, Taillard J, et al. CPAP treatment does not affect glucose-insulin metabolism in sleep apneic patients. Sleep Med. 2001;2(3):207-213. doi:10.1016/S1389-9457(00)00079-4

9. Harsch IA, Schahin SP, Radespiel-Troger M, et al. Continuous positive airway pressure treatment rapidly improves insulin sensitivity in patients with obstructive sleep apnea syndrome. Am J Respir Crit Care Med. 2004;169(2):156-162. doi:10.1164/rccm.200302-206OC

10. Czupryniak L, Loba J, Pawlowski M, et al. Treatment with continuous positive airway pressure may affect blood glucose levels in nondiabetic patients with obstructive sleep apnea syndrome. Sleep. 2005;28(5):601-603. doi:10.1093/sleep/28.5.601
11. Mastrototaro J. The MiniMed continuous glucose monitoring system (CGMS). J Pediatr Endocrinol Metab. 1999;12(Suppl3):751-758.

12. Wallace TM, Levy JC, Matthews DR. Use and abuse of HOMA modeling. Diabetes Care. 2004;27(6):1487-1495. doi:10.2337/ diacare.27.6.1487

13. Pallayova M, Donic V, Tomori Z. Beneficial effects of severe sleep apnea therapy on nocturnal glucose control in persons with type 2 diabetes mellitus. Diabetes Res Clin Pract. 2008;81(1):e8-e11. doi:10.1016/j.diabres.2008.03.012

14. Dawson A, Abel SL, Loving RT, et al. CPAP Therapy of Obstructive Sleep Apnea in Type 2 Diabetics Improves Glycemic Control during Sleep. J Clin Sleep Med. 2008;4(06):538-542. doi:10.5664/ jcsm. 27347

15. Shaw JE, Punjabi NM, Naughton MT, et al. The Effect of Treatment of Obstructive Sleep Apnea on Glycemic Control in Type 2 Diabetes. Am J Respir Crit Care Med. 2016;194(4):487-492. doi:10.1164/ rccm.201511-2260OC

16. Pamidi S, Wroblewski K, Stepien M, et al. Eight Hours of Nightly Continuous Positive Airway Pressure Treatment of Obstructive Sleep Apnea Improves Glucose Metabolism in Patients with Prediabetes. A Randomized Controlled Trial. Am J Respir Crit Care Med. 2015;192 (1):96-105. doi:10.1164/rcem.201408-1564OC

17. Drager LF, Brunoni AR, Jenner R, Lorenzi-Filho G, Benseñor IM, Lotufo PA. Effects of CPAP on body weight in patients with obstructive sleep apnoea: a meta-analysis of randomised trials. Thorax. 2015;70(3):258-264. doi:10.1136/thoraxjnl-2014-205361

18. Harsch IA, Schahin SP, Bruckner K, et al. The effect of continuous positive airway pressure treatment on insulin sensitivity in patients with obstructive sleep apnoea syndrome and type 2 diabetes. Respiration. 2004;71(3):252-259. doi:10.1159/000077423

19. Weinstock TG, Wang X, Rueschman M, et al. A controlled trial of CPAP therapy on metabolic control in individuals with impaired glucose tolerance and sleep apnea. Sleep. 2012;35(5):617-625. doi:10.5665/sleep. 1816

20. Muggeo M, Zoppini G, Bonora E, et al. Fasting plasma glucose variability predicts 10-year survival of type 2 diabetic patients: the Verona Diabetes Study. Diabetes Care. 2000;23(1):45-50. doi:10.2337/ diacare.23.1.45

21. Gimeno-Orna JA, Castro-Alonso FJ, Boned-Juliani B, et al. Fasting plasma glucose variability as a risk factor of retinopathy in Type 2 diabetic patients. J Diabetes Complications. 2003;17(2):78-81. doi:10.1016/S1056-8727(02)00197-6

22. Babu AR, Herdegen J, Fogelfeld L, et al. Type 2 diabetes, glycemic control, and continuous positive airway pressure in obstructive sleep apnea. Arch Intern Med. 2005;165(4):447-452. doi:10.1001/ archinte.165.4.447
International Journal of General Medicine

\section{Publish your work in this journal}

The International Journal of General Medicine is an international, peer-reviewed open-access journal that focuses on general and internal medicine, pathogenesis, epidemiology, diagnosis, monitoring and treatment protocols. The journal is characterized by the rapid reporting of reviews, original research and clinical studies across all disease areas. The manuscript management system is completely online and includes a very quick and fair peer-review system, which is all easy to use. Visit http://www.dovepress.com/ testimonials.php to read real quotes from published authors. 